\title{
Optically pumped lead-chalcogenide midinfrared lasers on Si substrates
}

\author{
Klaus Kellermann, Dmitri Zimin, Karim Alchalabi, Philippe Gasser, ${ }^{a}$ N. A. Pikhtin, ${ }^{\text {b) }}$ \\ and Hans Zogg ${ }^{\text {) }}$ \\ Thin Film Physics Group, Swiss Federal Institute of Technology, Zürich, Switzerland
}

(Received 17 July 2003; accepted 17 September 2003)

\begin{abstract}
Double heterostructure (DH) and quantum well (QW) $\mathrm{EuSe} / \mathrm{PbSe} / \mathrm{Pb}_{1-x} \mathrm{Eu}_{x} \mathrm{Se}$ edge-emitting laser structures on Si substrates are grown by molecular-beam epitaxy. They operate up to $250 \mathrm{~K}$ when pumped with $870 \mathrm{~nm}$ low-cost laser diodes with peak powers of $\sim 7 \mathrm{~W}$, and emit up to $200 \mathrm{~mW}$ peak output power at $\sim 5 \mu \mathrm{m}$ wavelength. Differential quantum efficiencies are up to $20 \%$. The threshold powers are limited by Shockley-Read recombination due to the high dislocation densities $\left(10^{8} \mathrm{~cm}^{-2}\right)$ in the active layers. Nearly similar maximum operation temperatures were observed when employing (111) - instead of (100) - oriented layers, as well when using QW rather than DH structures. Reduction of dislocation densities to $10^{7} \mathrm{~cm}^{-2}$ is feasible and will lead to nearly an order of magnitude lower threshold powers. (C) 2003 American Institute of Physics.

[DOI: $10.1063 / 1.1623924$ ]
\end{abstract}

\section{INTRODUCTION}

For a long time, the only diode laser sources for wavelengths above $3 \mu \mathrm{m}$ were electrically pumped leadchalcogenide (IV-VI) edge-emitting lasers. All of these lasers were fabricated on PbTe or PbSe bulk substrates. Such substrates are difficult to grow and handle, available in small sizes only, and exhibit very low thermal conductivity. The emission wavelengths up to $>20 \mu \mathrm{m}$ can be tuned by temperature, and, for ternary compounds like $\mathrm{Pb}_{1-x} \mathrm{Sn}_{x} \mathrm{Se}$, by composition $x$. The lasers have to be cryogenically cooled. For recent reviews, see Refs. 1 and 2. However, above room temperature (RT), lasing up to $60^{\circ} \mathrm{C}$ was reported for a $\mathrm{PbSe}$ double heterostructure (DH) in pulsed mode at $4.8 \mu \mathrm{m}^{3}$

Advantages in using IV-VI materials are the low Auger recombination and large tuning range. Auger recombination coefficients are up to 100 times lower than in III-V materials. The tuning range of IV-VIs is significantly higher ( $\sim 2 \times 10^{-3} / \mathrm{K}$ for multimode, $\sim 3 \times 10^{-4} / \mathrm{K}$ for monomode emission) compared to the quantum cascade lasers, an important aspect for spectroscopic applications. The IV-VI structures are rather tolerant to dislocations; it was even possible to fabricate distributed feedback lasers by just embossing a grating above the active region. ${ }^{4} \mathrm{It}$ is interesting to note that during the more than 30 years of development, an exact lattice match never dramatically improved laser performance. Threshold currents seem to be limited by other defects, at least at higher temperatures. In addition, quantum well (QW) structures did not yield the predicted significantly improved performances with respect to DH structures, contrary to what was found for III-V near-IR devices. This demonstrates that material quality is still limiting the behavior of IV-VI edge-emitting laser diodes.

${ }^{n}$ Swiss Federal Materials Testing and Research Laboratories EMPA, Dübendorf, Switzerland.

b) Ioffe Physico-Technical Institute, Semiconductor Luminescence and Injection Emitters Lab., St. Petersburg 194021, Russia.

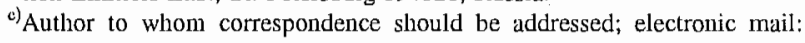
zogg@phys.ethz.ch
Renewed interest resulted recently in optically pumped vertical cavity surface-emitting laser (VCSEL) IV-VI structures on $\mathrm{BaF}_{2}(111)$ substrates. ${ }^{5,6}$ These VCSELs are presently illuminated at 1 to $2 \mu \mathrm{m}$ wavelength employing bulky pump lasers. This impedes their applications in low-cost systems. They operate pulsed even above RT. Again, no improvement in threshold power was found when going from DH to QW or even quantum dot structures; ${ }^{7} \mathrm{DH}$ structures showed the best performance. If such VCSELs are operated with subthreshold excitation, the emitted mid-IR radiation is a narrow band, the width is given by the finesse of the resonator structure. ${ }^{8}$

Surprisingly high structural quality, despite the large lattice and thermal expansion mismatch, is obtained for epitaxial lead-chalcogenide layers grown by molecular-beam epitaxy (MBE) on $\mathrm{Si}$ substrates. For compatibility, first, a $\mathrm{CaF}_{2}$ or a $\mathrm{CaF}_{2} / \mathrm{BaF}_{2}$ buffer layer is grown; followed by the IV-VI layer of a few $\mu \mathrm{m}$ thickness. We applied such layers for IR focal plane arrays for thermal imaging with cutoff wavelengths ranging from 4 to $12 \mu \mathrm{m}^{2,9}$ the Si substrate may even contain the read-out electronics. ${ }^{10}$ Dislocation densities are in the high $10^{6} \mathrm{~cm}^{-2}$ to low $10^{7} \mathrm{~cm}^{-2}$ range for layers with at least a few micrometer thickness grown on $\mathrm{Si}(111)$ substrates. Here, the thermal mismatch strain relaxes by dislocation glide in the main $\{100\}$-type glide planes, no cracks develop even for layers with thicknesses above 10 $\mu \mathrm{m}$. When grown on $\mathrm{Si}(100)$, the orientation of the IV-VI layer is (100), too. Dislocations can not glide in the main glide planes since the Schmid factors are zero. Here, the maximum thickness is limited to about $3 \mu \mathrm{m}$, above this value, the layers tend to crack. ${ }^{11}$

In the following, we describe optically pumped $\mathrm{DH}$ and QW edge-emitting IV-VI lasers grown mainly on $\mathrm{Si}(100)$ and $\mathrm{Si}(111)$ substrates. With optical pumping, no $p-n$ junctions with low ohmic contacts have to be fabricated, and design freedom is higher. This includes use of high resistance cladding layers like $\mathrm{Pb}_{1-x} \mathrm{Eu}_{x}$ Se with larger Eu-content, and lower carrier concentrations (which further diminishes Auger recombination). The laser structures are illuminated with 


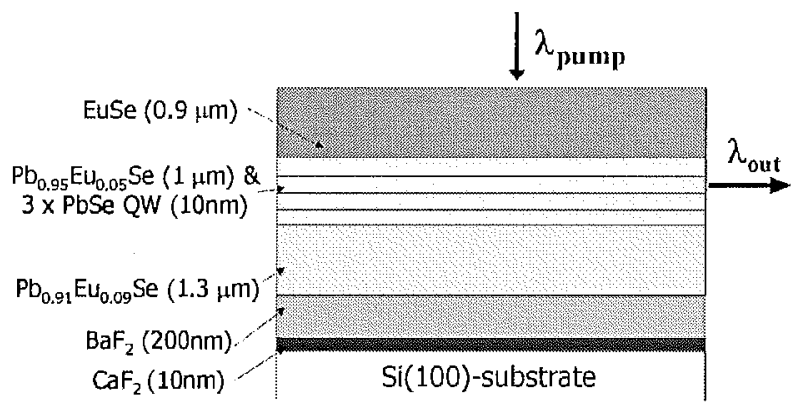

FIG. 1. Structure of an optically pumped QW IV-VI laser on a Si(100) substrate.

broad area (single stripe) high-power laser diodes emitting in the $850-1600 \mathrm{~nm}$ wavelength range. Such excitation is of special interest for compact and low-cost applications. More details may be found in Ref. 12. We compare the observed threshold power densities with theory, determine the dominant loss mechanism, and correlate it with the structural quality of the active layer.

\section{EXPERIMENT}

The structures are grown by solid-state $\mathrm{MBE}$ on $\mathrm{Si}(100)$ or $\mathrm{Si}(111)$ substrates. First, an epitaxial $\mathrm{CaF}_{2} / \mathrm{BaF}_{2}$ buffer layer is grown for compatibility. The $\mathrm{CaF}_{2}$ layer is typically 2 to $10 \mathrm{~nm}$, the $\mathrm{BaF}_{2}$ layer $200 \mathrm{~nm}$ thick. The structure of the QW edge-emitting laser of Fig. 1 grown on $\mathrm{Si}(100)$ consists of a bottom $\mathrm{Pb}_{1-x} \mathrm{Eu}_{x} \mathrm{Se}$ cladding layer, the active $\mathrm{Pb}_{1-y} \mathrm{Eu}_{y} \mathrm{Se}$ layer containing three $\mathrm{PbSe} \mathrm{QWs}$, and a top EuSe cladding layer which is transparent to the incoming pump beam. The $2-3 \mu \mathrm{m}$ thick IV-VI stack is (100) oriented. Alternatively, the active layer is just a $\mathrm{PbSe}$ or $\mathrm{PbTe}$ DH structure, with thicknesses between 100 and $400 \mathrm{~nm}$. The stack is lifted off from the substrate by dissolving the $\mathrm{BaF}_{2}$ buffer layer in water, cleaved into rectangles with sizes around one $\mathrm{mm}$ by $300 \mu \mathrm{m}$ (cleavage planes are $\{100\}$-type in lead chalcogenides), and clamped between a heatconducting substrate and a glass cover transparent to the pump beam (Fig. 2). Reflectivities of the cleaved mirrors are about $45 \%$ even without reflection coatings due to the high refractive indices.

The pump beam emitted from the exit mirror stripe of the III-V pump laser diode is focused onto the sample with cavity length $L$ as shown in Fig. 2 using a lens system. No lateral delineation is used (gain guided laser structure), the

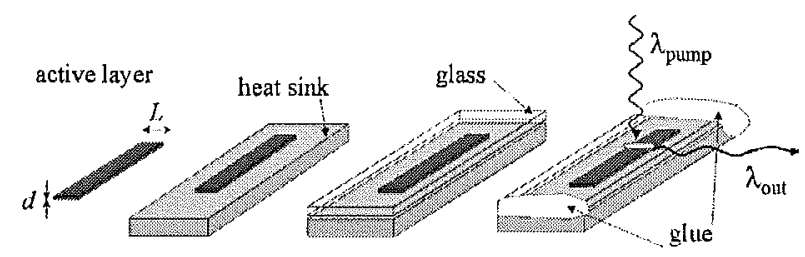

FIG. 2. Sample mount for epitaxial lifted off PbSe(100)-type laser stacks with cleaved side mirrors. The pump beam from the III- $V$ pump laser diode is focused to a stripe along the cavity length $L$ and forms the gain guided cavity.

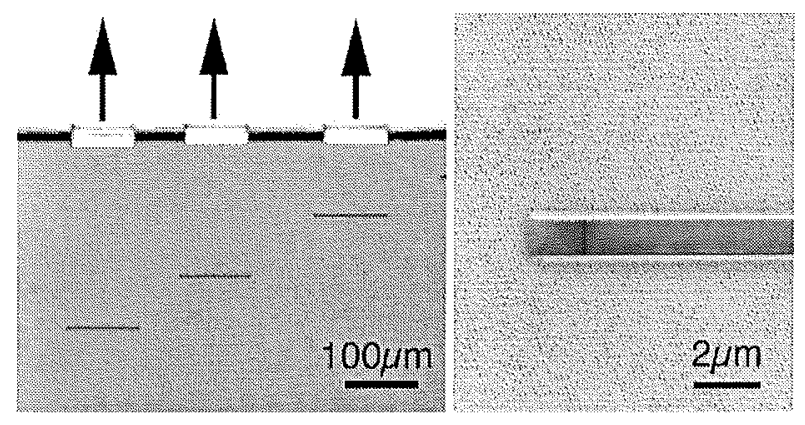

FIG. 3. Micrographic images (top views) of etched cavity mirrors for (111)oriented PbSe based stacks. Left-hand side: A sample containing three mirrors which form different cavity lengths $L$. The direction of the emitted light is indicated by arrows. Right-hand side: enlarged detail of a $\lambda / 4$ air groove back minor.

width is just determined by the width of the image of the pump laser emission face. Alternatively, to avoid the lens, the sample may just be placed several $\mu \mathrm{m}$ in front of the exit mirror of the pump laser source. A single stripe broad area pump laser diode emitting at $870 \mathrm{~nm}$ wavelength and a maximum pump peak power of 5 to $8 \mathrm{~W}$ at the sample were used for most experiments. Typical pulse widths were 150 ns with $8 \mathrm{kHz}$ repetition rate. In addition, we used a noncommercial pump laser emitting at $1.55 \mu \mathrm{m}$ for some measurements. ${ }^{12,13}$

Similar structures are grown on Si(111). Here, with (111) orientation, IV-VI stacks with improved structural quality result, and the thermal mismatch strains relax by glide of dislocations on each temperature cycle. ${ }^{9}$ Since the cleavage plane of IV-VIs is (100), the cavity mirrors have to be etched for (111)-oriented layers. We developed a dry processing technology based on focused ion beam etching which yields smooth vertical mirror faces. The back mirrors are just quarterwave $(\lambda / 4)$ grooves $(1.2 \mu \mathrm{m}$ wide) which yield $77 \%$ calculated reflectivity. A microscope image of a sample containing three mirror pairs forming different cavity lengths $L$ is shown in Fig. 3. The mirror reflectivities might be improved by etching additional $\lambda_{\text {air }} / 4$ grooves separated by a distance of $\lambda_{\text {medium }} / 4$. Etching one additional groove yields a calculated reflectivity of $98 \%$. However, as will be shown in Sec. V, lower mirror losses will not significantly improve threshold pump power densities for lengths $L$ of 300 $\mu \mathrm{m}$, as opposed to VCSELs where the highest reflectivity mirrors are required.

\section{EDGE-EMITTING OPTICALLY PUMPED IV-VI LASERS ON Si(100) SUBSTRATES}

The light-in versus light-out characteristics for the QW structure of Fig. I are shown in Fig. 4 (left-hand side) for different temperatures. Peak output power is up to $200 \mathrm{~mW}_{p}$ with the restricted pump power of $5.5 \mathrm{~W}_{p}$ in this case. Differential quantum efficiencies are as high as $20 \%$ (i.e., every fifth incoming photon is transformed into a lasing photon carrying about five times less energy), corresponding to a differential (power) efficiency of $4 \%$. These are rather high values for mid-IR emitters.

The laser emission is multimode (Fig. 4, right-hand side). No roll over of the differential quantum efficiencies 


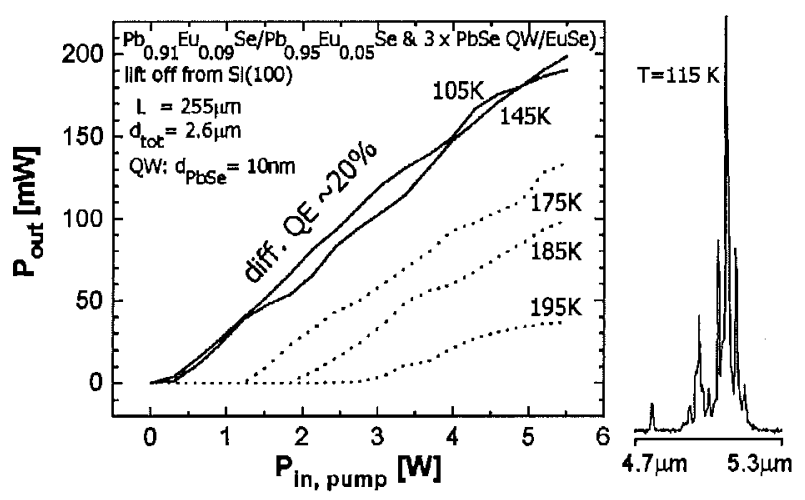

FIG. 4. Light-in/light-out characteristics of the PbSe QW laser structure of Fig. 1 [lifted off from the temporary $\mathrm{Si}(100)$ growth substrate] at different temperatures (left-hand side), and multimode spectrum at $115 \mathrm{~K}$ (right-hand side). The linewidths are limited by the spectrometer resolution.

occurs. The highest heat sink temperature where lasing is observed is about $240 \mathrm{~K}$ (restricted by the power of the pump laser). The threshold power as a function of temperature is shown in Fig. 5. The characteristic temperature $T_{0}$ is about $30 \mathrm{~K}$ [Fig. 5 curve (a)]. Still higher characteristic temperatures of $78 \mathrm{~K}$ were observed for similar laser structures grown on $\mathrm{Si}(100)$ and illuminated with the $870 \mathrm{~nm}$ pump laser [curve (b) in Fig. 5]. The highest characteristic temperature we obtained so far, as high as $122 \mathrm{~K}$, is observed when illuminating with the $1.55 \mu \mathrm{m}$ pump laser diode [curve (c) in Fig. 5]. Here, about two times less excess energy of the pump photons has to be absorbed. These $T_{0}$ are probably higher than previously obtained for any (electrically pumped) IV-VI laser diode in this temperature range. These high values are unexpected in view of the huge dislocation densities which might be higher than $10^{8} \mathrm{~cm}^{-2}$ in our films. RT operation seems possible by just increasing the intensity of the pump laser diode, and/or by somewhat further optimization of the structures including better thermal coupling to the heat sink. Up until now, we did not employ lateral confinement of the active layers, its width is just defined by the

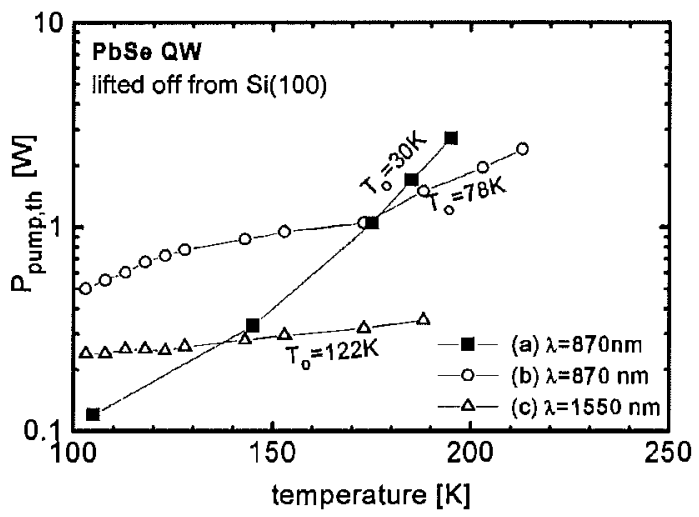

FIG. 5. Threshold pump powers as a function of temperature of (100)oriented laser structures lifted off from the $S$ i growth substrate. Laser structure of Fig. 4, illuminated with a $870 \mathrm{~mm}$ pump laser diode [curve (a)]. Laser structures grown on $\mathrm{Si}(100)$ with bigher characteristic temperatures $T_{o}$ illuminated with $870 \mathrm{~nm}$ (b) and $1550 \mathrm{~nm}$ (c) wavelengths.

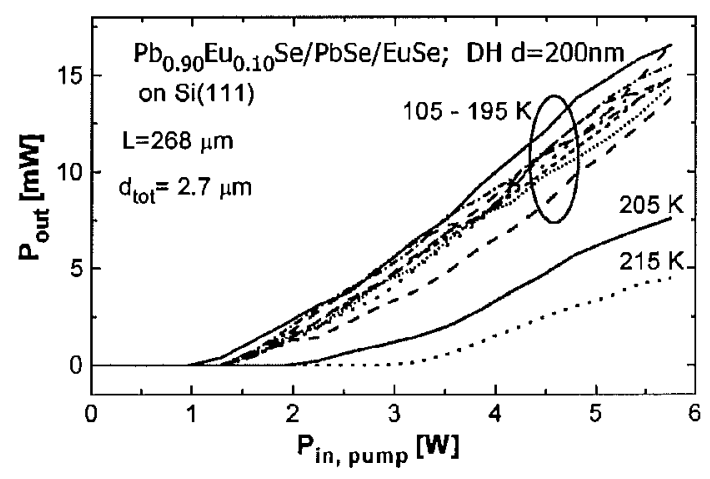

FIG. 6. Light-in/light-out characteristics of a (111) oriented PbSe DH laser structure grown on $\mathrm{Si}(111)$ with etched mirrors

width of the pump beam (gain guided cavity). In addition, threshold powers will diminish further if the structural quality of the active layer is increased (see Sec. VI).

For comparison, we fabricated (100)-oriented DH structures with the same method. Here, we obtained slightly lower maximum operation temperatures than with the QW structure described above. The low number of experimental data does, however, not allow us to draw sound quantitative conclusions.

\section{EDGE-EMITTING OPTICALLY PUMPED IV-VI LASERS ON Si(111) SUBSTRATES AND DETERMINATION OF THE INTRINSIC LOSS $\alpha_{i}$}

Here, we mainly used DH active layers instead of QW structures since QW designs showed a somewhat inferior performance for the (111) orientation. The mirrors are etched as described in Sec. II. No epitaxial lift off is needed, the structures remain on their original $\mathrm{Si}(111)$ substrate. Lightin/light-out diagrams of such a device are shown in Fig. 6 for different temperatures, and the threshold power as a function of temperature in Fig. 7. The threshold remains essentially constant up to $190 \mathrm{~K}$, then it increases. Lasing was observed up to $250 \mathrm{~K}$ heat sink temperature employing a pump laser diode delivering a peak power of about $7 \mathrm{~W}$ at the location of

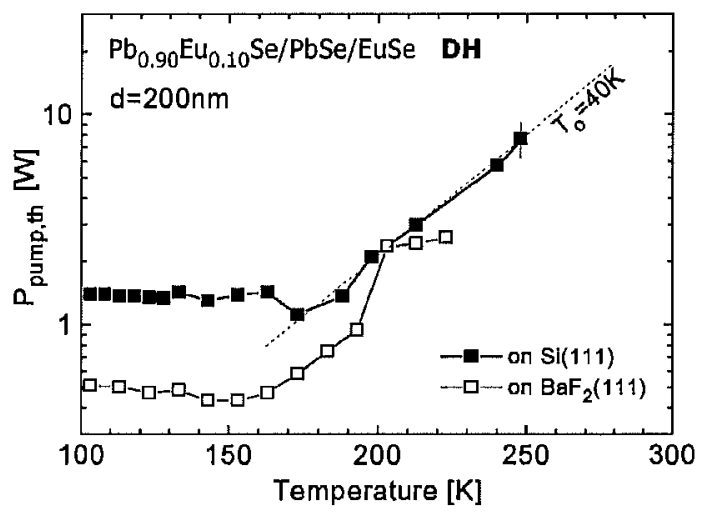

FIG. 7. Threshold pump powers as a function of temperature for a (111)oriented laser structure grown on $\mathrm{Si}(111)$ with etched mirror faces. For comparison, the results for a similar structure grown on $\mathrm{BaF}_{2}$ (showing lower threshold intensity at low temperatures) is included, too. 


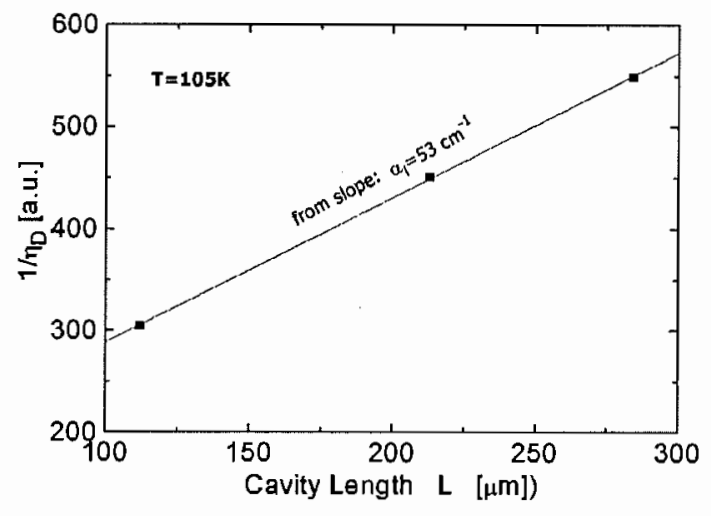

FIG. 8. Determination of the intrinsic loss $\alpha_{i}$ at $105 \mathrm{~K}$ from the slope of the inverse differential quantum efficiencies $\eta$ vs cavity lengths $L$.

the sample. Again, RT operation seems possible by just increasing the intensity of the pump laser diode, and/or by somewhat further optimization of the structures.

Employing different cavity lengths $L$, the internal loss $\alpha_{i}$ can be determined from the measured differential quantum efficiencies $\eta$

$$
\eta_{o} / \eta=1-2 \alpha_{i} / \ln \left(R_{1} R_{2}\right) \cdot L,
$$

where $\eta_{0}$ is the internal efficiency, and $R_{1}$ and $R_{2}$ the reflectivities of the front and back mirror, respectively. ${ }^{14}$ Differential efficiencies were determined employing the sample shown in Fig. 3 with three different cavity lengths $L$. Results are shown in Fig. 8 for a temperature of $105 \mathrm{~K}$.

The internal loss obtained from the slope of the curve is $\alpha_{i}=53 \mathrm{~cm}^{-1}$. This is a reasonable low value, but somewhat higher than free carrier absorption data. ${ }^{15}$ Free carrier absorption varies proportional to the temperature; therefore, at RT, a value below about $150 \mathrm{~cm}^{-1}$, is expected.

Nearly similar results, but with still lower threshold pump intensities at low temperatures are observed when the structures are grown on cleaved $\mathrm{BaF}_{2}(111)$ substrates. The threshold power as a function of temperature is plotted in Fig. 7 for comparison, too. With a $\mathrm{BaF}_{2}$ substrate, thermal expansion mismatch is absent since the thermal expansion coefficients of $\mathrm{BaF}_{2}$ and IV-VIs are nearly equal. However, the highest operation temperature we obtained is almost the same as that of the structures grown on Si(111) substrates. The curves show a nearly constant threshold power at low temperatures. This plateau was observed for some, but not all samples. However, when remeasuring several times, the plateau tilts toward a straighter line and with increased threshold power at the higher temperatures. No increased stability is observed for structures on $\mathrm{BaF}_{2}$ substrates compared to structures on Si.

\section{COMPARISON OF THRESHOLD INTENSITIES WITH THEORY}

For comparison with the experimental results, we calculated theoretical threshold intensities for a $\mathrm{DH}$ structure with thickness $d$ as follows. For lasing, the optical gain $g$ has to be larger than the losses

$$
g-\alpha_{i}-\alpha_{m}>0
$$

Here, $\alpha_{i}$ is the internal loss due to free carrier absorption and due to interface scattering and to defects. According to Sec. IV, $\alpha_{i}$ is expected not to exceed about $150 \mathrm{~cm}^{-1}$ even at RT by free carrier absorption and other possible loss mechanisms. The mirror loss is given by

$$
\alpha_{m}=-\ln \left(R_{\mathrm{l}} \cdot R_{2}\right) / 2 L,
$$

where $R_{1}$ and $R_{2}$ are the reflectivities of the front and back mirror, respectively, and $L$ is the cavity length. With $R_{1}$ $=0.45, R_{2}=0.78$, and $L=300 \mu \mathrm{m}, \alpha_{m} \approx 17 \mathrm{~cm}^{-1}$ is obtained. Compared to the high gains in $\mathrm{PbSe}$, mirror losses are small and not critical for long cavities, as opposed to VCSEL structures where very high $R$ are needed.

For the calculation, an injected carrier density provided by the pump laser $\delta n$ is chosen. From the total carrier dellsity $N=N_{a}+\delta n$ (we assumed a low equilibrium majority carrier concentration $N_{a}=10^{17} \mathrm{~cm}^{-3}$ ), the quasi Fermi levels for electrons $Q_{n}$ and holes $Q_{p}$ are calculated (full degenerate statistics have to be employed). A necessary condition for lasing is that the separation between $Q_{n}$ and $Q_{p}$ exceeds the band gap. To calculate the gain $g\left(Q_{n}, Q_{p}, T\right)$, explicit formulas are available ${ }^{15,16}$ for IV-VI DH structures. We used the material parameters for PbSe as given in Ref. 17. The injected carrier density $\delta n$ is now varied until $g-\alpha_{i}-\alpha_{m}$ is shightly larger than 0 . Since $g$ increases very steeply with $\delta n$, this is easily performed, and $\delta n$ does not change significantly even if the losses are somewhat higher than assumed. This now determines $\delta n$ at threshold.

The electronic losses are due to Auger recombination ( $\gamma_{\text {Auger }}$ is the Auger recombination coefficient), ShockleyRead recombination $N / \tau_{\mathrm{SR}}\left(\tau_{\mathrm{SR}}\right.$ is the Shockley-Read lifetime due to defects) and spontanous emission $R_{\mathrm{sp}}$. In steady state (neglecting temperature changes by Joule heating), the carrier density rate equation at threshold is ${ }^{14,18}$

$$
P_{\text {thresh }} /(d \cdot h \nu)=\gamma_{\text {Auger }} N^{3}+R_{\text {sp }}(N, T)+N / \tau_{\mathrm{SR}},
$$

where $P_{\text {thresh }}$ is the threshold power density $\left(\mathrm{W} / \mathrm{cm}^{2}\right)$ of the pump beam emitting photons with energy $h \nu$ (1.4 eV for 870 $\mathrm{nm}$ emission) and $d$ is the thickness of the DH. We assume that all radiation is completely absorbed in the PbSe DH. For $R_{\mathrm{sp}}$, we used the adapted results from Ref. 16. The Auger recombination coefficient $\gamma_{\text {Auger }}$ was originally calculated using nondegenerate statistics by Emtage, ${ }^{19}$ however, it does not change much for higher $N$ in the degenerate case. ${ }^{20,17}$ This is corroborated by time dependent recombination kinetics measurements of $\mathrm{PbSe}^{21}$ where an Auger coefficient of $1.1 \times 10^{-28} \mathrm{~cm}^{6} / \mathrm{s}$ was determined at RT and with excitation densities up to $3 \times 10^{18} \mathrm{~cm}^{-3}$. This is consistent with the values according to Emtage. For the Shockley-Read lifetime, typically $\tau_{\mathrm{SR}}=10 \mathrm{~ns}$ is assumed at low temperatures. ${ }^{15}$

Results are included in Fig. 9 as a function of temperature. The injected carrier densities needed at threshold are $\delta n=2 \times 10^{18} \mathrm{~cm}^{-3}$ at RT, $7 \times 10^{17} \mathrm{~cm}^{-3}$ at $200 \mathrm{~K}$, and 1.6 $\times 10^{17} \mathrm{~cm}^{-3}$ at $100 \mathrm{~K}$. The lowest threshold is obtained with low $N_{a}$ (assumed as $10^{17} \mathrm{~cm}^{-3}$ ). The steep increase of the Auger term toward RT which varies with $N^{3}$ is mainly due to the higher $\delta n$ needed for threshold with increasing tempera- 


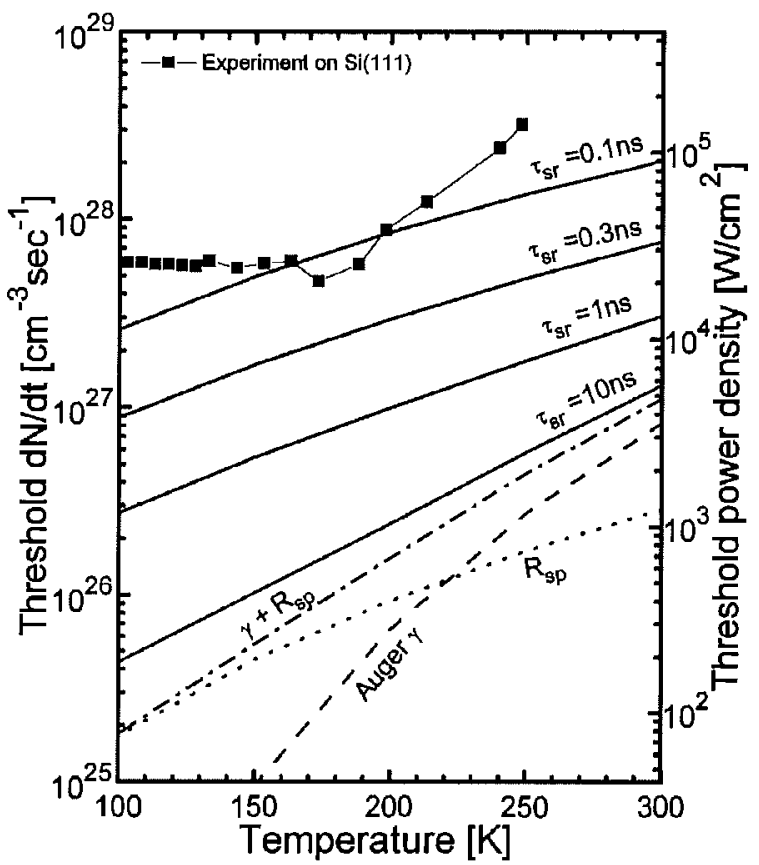

FIG. 9. Calculated threshold injection densities $d N / d t$ (left-hand side scale) and corresponding pump power densities for $870 \mathrm{~nm}$ pump wavelength (right-hand side scale) as a function of temperature for $\mathrm{PbSe} \mathrm{DH}$ with 200 $\mathrm{nm}$ thickness. The dashed and dotted lines are the contributions from Auger recombination $\gamma$ and spontaneous emission $R_{\mathrm{sp}}$. The full lines are the total losses including $\gamma, R_{\mathrm{sp}}$, and Shockley-Read recombination with lifetimes $\tau_{\mathrm{SR}}$ of $10 \mathrm{~ns}, 1 \mathrm{~ns}, 0.3 \mathrm{~ns}$, and $0.1 \mathrm{~ns}$. For sufficiently strained (111)-oriented DH structures (with only one $L$ valley populated due to the removed degeneracy) the total losses are up to three times lower. The experimental values are from the sample on Si(111) of Fig. 7.

ture, $\gamma_{\text {Auger }}$ does not depend much on temperature above 200 K. $R_{\text {sp }}$ dominates compared to the Auger term below $220 \mathrm{~K}$. However, the most important contribution is $\tau_{\mathrm{SR}}$. For $\tau_{\mathrm{SR}}$ $=10 \mathrm{~ns}$, this term dominates nearly up to RT. If shorter $\tau_{\mathrm{SR}}$ are assumed, they dominate even over the whole temperature range plotted. The curves are calculated for (100)-oriented structures where the four $\mathrm{L}$ valleys contribute equally to the gain.

The same results are obtained for (111) oriented layers if it is assumed that all four valleys are equally involved, as is true for unstrained DH. However, for (111) orientation, the degeneracy might be lifted by the strain. The valley perpendicular to the growth direction has lower energy than the three oblique valleys. This leads to lower threshold values if the separations of the energy levels are large enough. A gain calculation was performed for this orientation following the simplifications outlined in Ref. 24 assuming that only one L valley is involved, i.e., in the limit of very high strains in our case. This leads to significantly lower threshold illumination densities, typically up to three times lower than plotted in Fig. 9. However, the strain in our (111)-oriented DH is not known. If we assume that the bottom $\mathrm{Pb}_{1-x} \mathrm{Eu}_{x} \mathrm{Se}$ cladding layer is fully relaxed, the strain in the $\mathrm{PbSe} \mathrm{DH}$ is around 0.001 . This leads to an energy splitting of the order of $1 \mathrm{meV}$ between the perpendicular and oblique valleys, ${ }^{25,26}$ a value too small to expect a large effect on carrier populations near
RT. To benefit from the removed degeneracy of DH structures, the DH have to be designed for high internal strain.

To compare with the experimental results, one has to know the irradiated area of the samples. Since we did not perform a lateral delineation (gain guided structure), we estimated that the pump beam emitted from the exit mirror face of the pump laser diode is imaged as a $20 \mu \mathrm{m}$ wide stripe (see Fig. 2). With this estimate, the experimental results of the DH sample on $\mathrm{Si}(111)$ shown in Fig. 7 are plotted in the same figure. Clearly, a $\tau_{\mathrm{SR}}$ of $10 \mathrm{~ns}$ is much too long. Closer agreement with the experiment is obtained with $\tau_{\mathrm{SR}}$ as short as $0.1 \mathrm{~ns}$. Note that lateral carrier diffusion will lead to higher experimental values compared to a structure with a wave guide with additional lateral delineation (index guided cavity), and to which the theory is restricted.

\section{DISCUSSION}

Despite the deficiencies of the calculations above, we note that a rather short Shockley-Read lifetime around 0.1 ns roughly describes the threshold powers. To obtain lower thresholds and/or higher operating temperatures with the limited power delivered from our III-V pump laser diodes, the material quality, therefore, has to be improved. The typical threading dislocation density in our $0.2 \mu \mathrm{m}$ thick $\mathrm{PbSe} \mathrm{DH}$ is above $10^{8} \mathrm{~cm}^{-2}$. For thicker PbSe layers on $\mathrm{Si}(111)$ substrates (we employ for infrared detector focal plane arrays), we reproducibly obtain dislocation densities $\rho$ in the high $10^{6} \mathrm{~cm}^{-2}$ to low $10^{7} \mathrm{~cm}^{-2}$ range. ${ }^{9,10}$ We correlated this $\rho$ with the Shockley-Read lifetime obtained from the noise current densities in $p-n$ junction PbTe diodes, and found $1 / \tau_{\mathrm{SR}}$ proportional to $\rho$. A lifetime $\tau_{\mathrm{SR}}$ close to $1 \mathrm{~ns}$ was deduced from these measurements. ${ }^{10}$ If we assume that $\tau_{\mathrm{SR}}$ in these $p-n$ junctions at low carrier concentrations is the same as that responsible for the losses in the laser structures, and knowing $\rho \sim 10^{8} \mathrm{~cm}^{-2}$ for the DH structure, a lifetime $\tau_{\mathrm{SR}}=0.1 \mathrm{~ns}$ results. This is consistent with the threshold intensity calculations and the present measurements.

To further improve our laser structures, one, therefore, has to reduce the threading dislocation densities. According to our results with thicker layers for IR detectors on Si substrates, it will be possible to decrease the dislocation density in the $200 \mathrm{~nm}$ thin DH structure to $10^{7} \mathrm{~cm}^{-2}$, for example, by using a more sophisticated cladding layer with graded composition design. This will lead to longer $\tau_{\mathrm{SR}}$ up to $\sim 1 \mathrm{~ns}$ with correspondingly reduced threshold intensities and, therefore, to considerably higher operation temperatures.

DH structures with (111) orientation did not yet lead to tremendously improved performance as compared to the (100) orientation, as expected from the higher structural quality of layers grown on $\mathrm{Si}(111)$. In addition, to take advantage of the removed four-fold degeneracy at the L-valley band extrema in the (111) orientation, the DH have to be designed in a manner that they are highly strained.

It is interesting to note that so far we did not get substantially improved results with QW compared to DH structures on $\mathrm{Si}$ as well as on $\mathrm{BaF}_{2}$ substrates. Such an improvement is expected due to the lower carrier densities needed to get inversion ${ }^{22}$ and decreased Auger recombination rates for 
low- $d$ structures. ${ }^{23}$ As already mentioned, similar observations have been made for (100)-oriented electrically pumped QW and DH lasers ${ }^{1,2}$ on IV-VI substrates, and optically pumped (111)-oriented VCSELs grown on $\mathrm{BaF}_{2} .{ }^{7}$ For the latter case, the Eu-containing host layer might be responsible for this due to a significant decrease in carrier lifetimes when adding $\mathrm{Eu}$ to $\mathrm{PbSe}$. Recently, in an investigation of photoluminescence intensities, the decrease was attributed to intermixing of Euf $f 4$ states with the valence band. ${ }^{27}$

\section{CONCLUSIONS}

Epitaxial lead-chalcogenide on Si structures show reasonable lasing properties despite a high dislocation density of up to above $10^{8} \mathrm{~cm}^{-2}$. DH and QW structures lase up to $\sim 250 \mathrm{~K}$ when pumped optically with low-cost III-V laser diodes with about 5 to $8 \mathrm{~W}$ peak power, and differential quantum efficiencies are up to as high as $20 \%$.

Due to the ease of fabrication, the realized edge-emitting lasers allow application even for low-cost spectroscopic gas sensing of common gases like $\mathrm{CO}_{2}, \mathrm{CO}$, or $\mathrm{CH}_{4}$. RT operation will be possible by somewhat improved material quality and improved layout including index guided structures.

Higher operation temperatures seem easily possible just by increasing the pump laser intensity at the sample surface, no roll over was observed up until now. A better solution is to decrease dislocation densities. A decrease to at most $10^{7} \mathrm{~cm}^{-2}$ (as is typical for the material we use for fabrication of infrared detector arrays) is experimentally feasible. This would lead to about ten times longer Shockley-Read lifetimes $\tau_{\mathrm{SR}}$ which mainly determine the threshold power. From this, a significant increase of the maximum operating temperature is expected according to the theory, i.e., RT operation would result even with moderate pump illumination conditions.

To obtain full benefits from QW structures, i.e., still lower threshold excitation as compared to $\mathrm{DH}$ structures, the quality of the electrically active layers has to be significantly improved, too. Ternary host layers containing the PbSe QW have to be adapted which exhibit high enough carrier lifetimes. The $\mathrm{Pb}_{1-x} \mathrm{Eu}_{x} \mathrm{Se}$ chosen for this purpose by several groups seems not to be the best choice probably due to intermixing of Eu $f 4$ states with the valence band.

\section{ACKNOWLEDGMENT}

The work was sponsored by the Swiss National Foundation and the GR-Stiftung.

${ }^{1}$ M. Tacke, Philos. Trans. R. Soc. London, Ser. A 359, 547 (2001).

${ }^{2} \mathrm{H}$. Zogg and A. Ishida, in Infrared Detectors and Emitters, edited by P. Capper and C. T. Elliott (Kluwer, Dordrecht, 2000).

${ }^{3}$ U. P. Schiessel and J. Rohr, Infrared Phys. Technol. 40, 325 (1999).

${ }^{4}$ M. A. Fach, H. Böttner, K.-H. Schlereth, and M. Tacke, IEEE J. Quantum Electron. 30, 235 (1994).

${ }^{5}$ W. Heiss, T. Schwarzl, G. Springholz, K. Biermann, and K. Reimann, Appl. Phys. Lett. 78, 862 (2001).

${ }^{6}$ F. Zhao, H. Wu, Lalith Jayasinghe, and Z. Shi, Appl. Phys. Lett. 80, 1129 (2002).

${ }^{7}$ J. Fürst, H. Pascher, T. Sclıwarzl, M. Böberl, W. Heiss, G. Springholz, and G. Bauer, Appl. Phys. Lett. 81, 208 (2002).

${ }^{8}$ K. Kellermann, D. Zimiu, K. Alchalabi, N. A. Pikhtin, and H. Zogg, 1EE Proc.: Optoelectron. , (2003) (to be published).

${ }^{9}$ H. Zogg, in Optoelectronic Properties of Semiconductors and Superlattices, Lead Chalcogenides: Physics and Applications Vol. 18, edited by D. Khokhlov (Taylor and Francis, New York, 2003), pp. 587-616.

${ }^{10}$ H. Zogg, K. Alchalabi, D. Zimin, and K. Kellermann, IEEE Trans. Electron Devices 50, 209 (2003).

${ }^{11}$ P. Müller, A. Fach, J. John, A. N. Tiwari, H. Zogg, and G. Kostorz, J. Appl. Phys. 79, 1911 (1996).

${ }^{12}$ K. Kellermann, Ph.D. thesis, 2003, (in preparation).

${ }^{13}$ E. G. Golikova, V. A. Kureshov, A. Yu. Leshko, A. V. Lyutetskii, N. A Pikhtin, Yu. A. Ryaboshtan, G. A. Skrynnikov, I. S. Tarasov, and Zh. I. Alferov, Semiconductors 34, 853 (2000).

${ }^{14}$ G. P. Agraval and N. K. Dutta, Semiconductor Lasers, 2nd ed. (Kluwer, Dordreclit, 1993).

${ }^{15}$ W. W. Anderson, IEEE J. Quantum Electron. 13, 532 (1977).

${ }^{16}$ F. Galeskii, A. E. Yunovich, K. H. Hermann, H. Kostial, I. Rechenberg, and P. Schäfer, Phys. Status Solidi B 88, 675 (1978).

${ }^{17} \mathrm{H}$. Zogg, W. Vogt, and W. Baumgartner, Solid-State Electron. 25, 1147 (1982).

${ }^{18}$ S. Khosravani and Z. Shi, Appl. Phys. Lett. 78, 139 (2001).

${ }^{19}$ P. R. Emtage, J. Appl. Phys. 47, 2565 (1976).

${ }^{20}$ O. Ziep, D. Genzow, M. Mocker, and K. H. Herrmann, Phys. Status Solidi B 99, 129 (1980).

${ }^{21}$ R. Klann, T. Höfer, R. Buhleier, T. Elsässer, and J. W. Tomm, J. Appl. Phys. 77, 277 (1995).

${ }^{22}$ G. Bauer, M. Kriechbaum, Z. Shi, and M. Tacke, J. Nonlinear Opt. Phys. Mater. 4, 283 (1995)

${ }^{23}$ F. Lemke and M. Mocker, Phys. Status Solidi B 167, 219 (1991).

${ }^{24}$ A. Majumdar, H. Z. Xu, S. Khosravani, F. Zhao, L. Jayasinghe, and Z. Shi, Appl. Phys. Lett. 82, 493 (2003).

${ }^{25} \mathrm{~J}$. Singleton, E. Kress-Rogers, A. V. Lewis, R. J. Nicholas, E. J. Fantner, G. Bauer, and A. Otero, J. Phys. C 19, 77 (1986).

${ }^{26} \mathrm{C}$. Maissen, Ph.D. thesis, 1992.

${ }^{27}$ M. Böberl, W. Heiss, T. Schwarzl, K. Wiesauer, and G. Springholz, Appl. Phys. Lett. 82, 4065 (2003). 